\title{
De l'Armistice Day au Tsunami Sïlence : refonte du silence comme outil de construction mémorielle
}

Le 8 novembre 2015, à l'occasion du Remembrance Sunday au Royaume-Uni, se sont tenues les commémorations de la fin de la Première Guerre mondiale.

L'auteur : Lucas FOESTER est titulaire d'un master en science politique.

Plusieurs membres de la famille royale et du gouvernement se sont succédés devant le cénotaphe à Whitehall pour y déposer les traditionnelles couronnes de coquelicots.

Comme le veut l'usage, deux minutes de silence ont été observées à travers le Royaume-Uni afin de commémorer les épreuves endurées par le pays et ses citoyens. Il faut remonter jusqu'à 1919 pour saisir les origines et les objectifs de ce silence qui marque véritablement une pause au cours de laquelle toute activité cesse sur tout le territoire du Royaume-Uni. Si cet usage a perduré jusqu'à devenir un des éléments centraux de la culture commémorative britannique, Andy McSMITH a pointé une augmentation du recours à la minute de silence comme outil de commémoration au cours des dernières années. Les attentats du 11 septembre 2001, ceux de Madrid en 2004 et de Londres en 2005, ou encore les tsunamis en Asie sont autant d'événements commémorés par le silence qui confirment ce que le journaliste décrit comme une « inflation du silence »'.

La présente contribution se fixe deux objectifs. Dans un premier temps, il s'agit d'étudier la mise en place des deux minutes de silence au Royaume-Uni et de comparer la signification que lui ont assignée les autorités publiques avec le sens, potentiellement différent, que lui attribuaient d'autres catégories d'acteurs. Dans un second temps, ce travail propose une étude comparative entre les deux minutes de silence de l'Armistice et les trois minutes observées en hommage aux victimes des tsunamis. II s'agit de mettre en évidence la manière dont la forme de cet outil de construction mémorielle a été récupérée alors que le fond a été modifié de façon importante.

'MCSMITH Andy, "A brief history of silence: When no noise is good noise ", The Independent, disponible à l'adresse suivante : http://www.independent.co.uk/ (consultée le 10 novembre 2014). 


\section{Précisions sur le concept de silence}

\section{Quelle interaction entre silence et mémoire ?}

Dans son acception classique, le terme «silence » a plusieurs sens. D'abord, il signifie logiquement une absence de bruit'2. Dans le champ de la psychologie sociale, il traduit le fait de se taire, de ne pas parler, définition à laquelle se raccroche le concept de "mutisme $»^{3}$. Enfin, dans le langage musical, le silence se conçoit comme l'interruption volontaire du son sur une durée déterminée ${ }^{4}$.

Il est intéressant de constater que la technique commémorative de la minute de silence emprunte aux trois significations du silence.

En effet, au cours d'une minute de silence, il est possible d'identifier une brève absence de bruit, une forme de mutisme des individus y participant, et le caractère volontaire semble inhérent à ce genre de manifestation.

La mémoire ne s'oppose pas à l'oubli5. Comme le dit Tzvetan TODOROV : «[...] elle est ce processus incessant de tri et, donc d'oubli $"{ }^{6}$. Cet auteur va jusqu'à considérer l'oubli comme une condition fondamentale du processus mémoriel7. Dans le même ordre d'idée, il est vain de vouloir opposer le silence à la mémoire, même si le premier ne conditionne pas la seconde. Dès lors, le postulat de cette contribution consiste à affirmer que le silence peut intervenir dans la "création » de mémoire.

Le développement du silence commémoratif dans le cadre des célébrations de l'Armistice au Royaume-Uni est un exemple pertinent du rôle d'outil de construction mémorielle que peut endosser le silence.

\footnotetext{
${ }^{2}$ Coman Alin et StONE Charles, "Toward a science of silence : The consequences of leaving a memory unsaid ", Perspectives on Psychological Science, 2012, vol. 7, p. 43

3 Ibid., p. 44

${ }^{4}$ "Le son du silence », Spirale, 2001, n 17, p. 167.

${ }^{5}$ TODOROv Tzvetan, Les abus de la mémoire, Paris, Arléa, 2004, p. 14.

${ }^{6}$ RIoux Jean-Pierre, «Devoir de mémoire, devoir d'intelligence », Vingtième Siècle. Revue d'histoire, 2002 n० 73 , p. 163

${ }^{7}$ MAYER Françoise et VASICEK Zdenek, "Le passé et le présent, la mémoire et l'histoire ", in MAUREL MarieClaude et MAYER Françoise (dir.), L'Europe et ses représentations du passé. Les tourments de la mémoire, Paris, L'Harmattan, coll. « Logiques sociales », 2008, p. 176
} 


\section{Comparer les silences}

Comparer les recours au silence n'est pas un exercice courant. Comme dans tout exercice de comparaison, il est impératif de s'assurer que ce qui est comparé est comparable. Si le choix de comparer le silence de l'Armistice et le silence en hommage aux victimes du tsunami peut être critiqué, il est, néanmoins, justifiable sur la base de plusieurs éléments.

Tout d'abord, ces deux commémorations par le silence font suite à un événement marquant qui a causé un grand nombre de victimes. Le fait que ces deux "pauses " commémoratives se soient propagées jusque dans les écoles, les stations de métro ou encore les bureaux est également un caractère distinctif. Ensuite, ces deux silences sont le fait des autorités, et non d'un élan de spontanéité. À la différence de certaines minutes de silence organisées dans l'intimité d'un stade de football pour rendre hommage à un supporter ou à un joueur disparu, les deux silences analysés ont, eux, fait l'objet d'une planification par les autorités. Enfin, la comparaison entre le silence de l'Armistice et celui en hommage aux victimes du tsunami est pertinente du fait que les deux manifestations sont basées sur la communion plutôt que sur la différenciation. En effet, certains recours récents au silence commémorent des attaques terroristes. Ces silences sont généralement accompagnés de discours insistant sur la différence qui existe entre " eux » et "nous ». Les participants sont unis dans le rejet d'actes qu'ils ne cautionnent pas. Le Tsunami Silence, lui, à l'instar du silence de l'Armistice, vise à unir tous les participants afin que chacun partage la peine des familles endeuillées.

\section{Le recours à la minute de silence}

Étant donné que la première partie de cette contribution est axée sur les deux minutes de silence observées lors des cérémonies de l'Armistice au Royaume-Uni, cette section propose un court historique de la façon dont cette manifestation est devenue un élément-clé des commémorations du 11 novembre outre-Manche.

Sir Percy FitzPATRICK, ancien représentant de la couronne en Afrique du Sud pendant la Guerre des Boers, fut celui qui proposa que les commémorations se tiennent le jour du cessez-le-feu. II proposa également d'avoir recours au silence en se basant sur l'expérience acquise en Afrique du Sud. Le mémorandum remis par Sir Percy FITZPATRICK au Gouvernement stipulait que : 
movement were suspended for three minutes that we might concentrate as one in thinking of those - the living and the dead - who had pledged and given themselves for all that we believe in ${ }^{8}$.

Il est intéressant de noter que le projet fut très vite avalisé par le Roi George v. Néanmoins, la décision officielle n'étant intervenue que le 7 novembre 1919, les quotidiens britanniques furent chargés de placer des «rappels" sur leur couverture le jour des commémorations?.

Ce n'était cependant pas la première fois qu'on avait recours au silence pour des commémorations au Royaume-Uni puisque la mort du Roi Edward VII en 1910 ainsi que le naufrage du Titanic en 1912 avaient été salués par une minute de silence ${ }^{10}$. Cependant, ces manifestations n'eurent lieu qu'une seule fois. Le succès du « premier silence " fut tel qu'après deux ans, en 1921, la plupart des participants considéraient cette courte de pause annuelle comme sacrée". Néanmoins, il serait erroné de présenter les deux minutes de silence comme un événement accueilli favorablement par l'ensemble des citoyens britanniques. La section suivante sera consacrée à l'étude des sens différents, des diverses interprétations données au silence lors des célébrations de l'Armistice.

\section{The Armistice Day et les deux minutes de silence}

\section{La nécessité de créer l'unité}

Plusieurs éléments permettent de comprendre l'empressement de l'autorité étatique à mettre en place toute une série de commémorations dès 1919. Tout d'abord, les commémorations sont, pour le gouvernement britannique, un outil de légitimation.

Seul le vainqueur (ou l'un des vainqueurs) souhaite se souvenir d'un conflit tel que la Première Guerre mondiale. Ensuite, l'État est inquiet depuis la Révolution russe de $1917^{12}$.

\footnotetext{
${ }^{8}$ Traduction proposée par l'auteur de l'article : «Pendant la guerre, nous, en Afrique du Sud, avons observé ce que nous appelons la 'pause des trois minutes'. Chaque jour, à midi, tous les travaux, tous les discours et tous les mouvements étaient suspendus durant trois minutes pour que nous puissions nous unir dans la pensée de ceux - vivants ou morts - qui avaient souffert et donné leur vie pour tout ce en quoi nous croyons ". GREGORY Adrian, The Silence of Memory: Armistice Day 1919-1946, Oxford, Berg Publishers, 1994, p. 9. - GREGoRY Adrian, The Silence of Memory: Armistice Day 1919-1946, op. cit., p. 11.

${ }^{10}$ BRown Steven D., "Three minutes of silence : Social technologies of public commemoration ", Theory \& Psychology, 2012, vol. 22, p. 243

"GREGORY Adrian, The Silence of Memory: Armistice Day 1919-1946, op. cit., p. 17.

${ }^{12}$ Ibid., p. 65.
} 
Le bruit court que les factions d'extrême-gauche et d'extrême-droite, considérées comme des menaces par l'État, recrutent des vétérans en nombre. D'ailleurs, dans quel état d'esprit reviennent ces vétérans? Les mutineries au moment de la démobilisation sont un fait, et le Gouvernement britannique sait que les premiers instants de la période post-conflit vont être cruciaux. Enfin, la stratégie la plus efficace mise en place par le pouvoir étatique fut la création d'un langage du sacrifice ${ }^{13}$. Le terrible coût de la guerre est considéré comme un sacrifice nécessaire afin qu'un tel événement ne puisse plus se reproduire.

\section{Le parti pris de la mémoire officielle}

Si les autorités publiques ont rapidement mis en place toute une série de commémorations en rapport avec la Première Guerre mondiale, chaque belligérant a dû poser un choix afin de décider de la façon dont le conflit serait retenu ainsi que de la nature du sacrifice qui serait retenu comme central. Au Royaume-Uni, c'est le sacrifice des familles qui fut mis en avant ${ }^{14}$. Toute une panoplie d'aides furent apportées à ces familles endeuillées dont un ou plusieurs membres avaient péri. De cette manière, les autorités publiques pensaient limiter le nombre d'opinions dissidentes relatives à la guerre sans pour autant imposer une vision unilatérale. En effet, derrière les possibles désaccords, tous les individus s'uniraient dans le souvenir des victimes du conflit ${ }^{15}$.

Cependant, la colère grondait parmi les vétérans. Démobilisés sans fanfares, ils rentrèrent au pays riches d'une solde qui ne suffisait pas à leur assurer un niveau de vie correct. Dès lors, les anciens combattants se mirent en quête d'un emploi.

Mais, peu qualifiés à cause de leur jeune âge lors de l'enrôlement ou diminués à cause d'une guerre qui a inventé la destruction des corps, les vétérans composèrent bientôt la majorité des sans-emploi au Royaume-Uni. La situation atteint un point tel que les termes "sans-emploi " et "vétérans " finirent par devenir des synonymes ${ }^{16}$. De plus, en choisissant d'encourager les employeurs à embaucher des vétérans, mais sans intervention légale, tout en refusant d'instaurer des mesures de soutien public pour aider ceux qui n'étaient plus en mesure de travailler ${ }^{17}$, les autorités publiques ont, semble-t-il, tenté de réduire les anciens combattants au silence.

\footnotetext{
${ }^{13}$ Gregory Adrian, The Silence of Memory: Armistice Day 1919-1946, op. cit., p. 23

${ }^{14} \mathrm{Ibid} .$, p. 226.

${ }^{15} \mathrm{lbid}$.

${ }^{16} \mathrm{Ibid}$., p. 54 
Livrés à eux-mêmes, les vétérans se mirent en tête de contester cette primauté accordée à la souffrance des familles. Mais, entretemps, les deux minutes de silence décrétées par le pouvoir étaient déjà tellement ancrées dans la tradition commémorative britannique qu'essayer d'en transformer la signification eût été contre-productif.

Néanmoins, cet état de fait illustre bien la transition que Johann MICHEL a théorisée, entre centralisme mémoriel et gouvernance mémorielle ${ }^{18}$. Rapidement après la Première Guerre mondiale, les autorités publiques perdent leur position dominante dans le processus de construction mémorielle. Certains acteurs non-étatiques développent toute une série de mémoires dites locales et, on peut, dès lors, parler de politique mémorielle négociée ${ }^{19}$. En effet, des mémoriaux sont érigés dans la plupart des comtés et diocèses pour satisfaire un besoin de "nommer les morts ". C'est pourquoi la plupart de ces monuments consistent en des séries de noms qui donnent à la souffrance une dimension plus intime car limitée géographiquement.

Dès lors, sans avoir à se rétracter, les autorités publiques n'ont eu qu'à accueillir les anciens combattants lors des commémorations, leur présence servant de rappel à la population de la dette contractée à leur égard. Ce compromis devait satisfaire toutes les parties et aucune à la fois. Les autorités publiques, si elles ont géré cette crise, ont dû faire face à celle du langage du sacrifice à l'entame de la Deuxième Guerre mondiale. Quant aux vétérans, ils ne furent jamais certains que le sens des commémorations de novembre s'était ouvert à eux.

Cependant, malgré une histoire marquée par les négociations et les rapports conflictuels, la pause des deux minutes observée au Royaume-Uni a conservé cette capacité à rassembler les citoyens. Ainsi, tandis que chaque Britannique, lorsque le silence est rompu, reprend son propre chemin, la communauté toute entière continue d'avancer dans le même sens.

\section{Au nom de la justice sociale : The Haig Fund Appeal}

Les commémorations ont, dans ce cas précis, traduit une « reconnaissance de dette " envers les combattants (tombés ou survivants) ${ }^{20}$. Cependant, de nombreuses manifestations furent nécessaires avant que la collectivité ne comprenne qu'il fallait " payer " cette dette.

\footnotetext{
${ }^{18}$ MiCHEL Johann, Gouverner les mémoires. Les politiques mémorielles en France, Paris, Presses Universitaires de France, 2010, pp. 49-50..

${ }^{20}$ GREGORY Adrian, The Silence of Memory: Armistice Day 1919-1946, op. cit., p. 58
} 
Parmi tous les actes forts posés par les vétérans pour traduire le sentiment de trahison, retenons l'abandon de médailles de guerre et les défilés devant le cénotaphe de Whitehall dont l'un d'eux est défini en ces termes :

\begin{abstract}
[...] Whitehall and the streets surrounding it were densely packed with mourners and sight seers. This time they saw a sight that they had never seen before. As the banners of the various contingents were raised ready for the march, it was seen that each banner had hundreds of thousands of ex-service men's medals pinned on to it. Thousands of ex-servicemen took pawn tickets from their pockets and pinned them on the lapels of their coats. The bands of the unemployed movement were draped in red and black, and at the head of our procession was carried a large wreath with an inscription that read: From the living

victims - the unemployed - to our dead comrades who died in vain ${ }^{21}$.
\end{abstract}

Les sacrifices consentis par les vétérans finiront par trouver une place dans les commémorations à travers l'appel de fonds du Maréchal de la British Legion, Douglas HAIG ${ }^{22}$. Celui-ci décida, en 1921, d'entamer la distribution de coquelicots artificiels en échange d'une donation. Importés de France dans un premier temps, les coquelicots seront rapidement fabriqués en série par des vétérans dans une usine rachetée par la Légion britannique.

L'argent collecté au niveau local est centralisé au quartier général de la British Legion avant d'être redistribué aux entités locales en fonction des besoins ${ }^{23}$. Cette action visait à venir en aide aux vétérans sans revenus, aux handicapés et blessés de guerre ainsi qu'aux familles dépendantes de ceux qui avaient été tués ou gravement blessés sur le champ de bataille. L' " appel du coquelicot " a été, depuis sa création, un énorme succès et est aujourd'hui, au même titre que les deux minutes de silence, un élément-clé des commémorations de novembre.

\title{
L'échec de la « Der des Ders ॥
}

Comme cela a été développé précédemment, l'une des stratégies mises en place par le Gouvernement britannique pour donner un sens au massacre de la Première Guerre mondiale fut d'interpréter ce dernier comme un sacrifice.

${ }^{21}$ Traduction proposée par l'auteur de l'article : « Whitehall et les rues voisines étaient remplies de pleureuses et de passants. Cette fois, ils assistèrent à un spectacle inédit. Lorsque les bannières des différents contingents militaires se dressèrent, on put voir que des centaines de milliers de médailles de vétérans avaient été fixées sur les étoffes. Des milliers d'anciens combattants avaient épinglé leurs reconnaissances de dettes sur leurs manteaux. Drapés de rouge et de noir, les sans-emploi avaient placé en tête de procession une couronne de fleurs avec une inscription sur laquelle on pouvait lire : «De la part des victimes toujours en vie - les sans-emplois - à nos camarades morts en vain ». GREGORY Adrian, The Silence of Memory: Armistice Day 1919-1946, op. cit., p. 59. ${ }^{22}$ GrEGORY Adrian, The Silence of Memory: Armistice Day 1919-1946, op. cit., p. 93 ${ }^{23}$ Ibid. 
Un sacrifice qui avait du sens puisqu'il avait servi à contrecarrer les désirs expansionnistes de l'Empire allemand. Mais, progressivement, ce sens donné à la disparition de tant de Britanniques fut trouvé moins évident, et prit davantage la forme d'une exhortation morale ${ }^{24}$.

La signification des cérémonies de l'Armistice ainsi que la mémoire du sacrifice "pour que cela ne se reproduise jamais » entrent en crise dès le milieu des années 1930. La montée du fascisme et du nazisme semblent mener inévitablement à un nouveau conflit. Lorsque la Deuxième Guerre mondiale éclate, les conscrits partent au combat en étant désormais convaincus que leur sacrifice ne servira à rien ${ }^{25}$.

À partir de 1945, les commémorations s'articuleront sur la base des mémoires des deux conflits mondiaux. Mais la conviction selon laquelle ces tueries ont été des sacrifices utiles qui ont débouché sur un monde meilleur ne trouve plus d'écho auprès de ceux qui souffrent. Désormais, les deux minutes de silence sont une marque de respect envers les victimes de la guerre. Mais peut-être se tait-on également pour rendre hommage à la disparition du concept de sacrifice.

\section{Les récentes commémorations par le silence : continuité ou rupture?}

La présente section a pour objectif de présenter plusieurs éléments qui différencient les deux minutes de silence au Royaume-Uni de la pause des trois minutes observées à travers le monde en hommage aux victimes des tsunamis de 2005. Ces différences ont trait tant à la forme qu'au fond de ces manifestations. Une attention particulière est portée à l'utilisation différente qui est faite du silence ainsi qu'à son rôle variable selon qu'il s'agit de l'une ou de l'autre commémoration.

\section{Présence ou absence des commémorés?}

Les deux minutes de silence observées au Royaume-Uni à partir de 1919 sont basées sur l'absence des commémorés ${ }^{26}$. Bien qu'à la suite de choix politiques, l'accent fut mis sur la souffrance des familles, l'objectif premier était de saluer le sacrifice que des centaines de milliers de Britanniques avaient consenti afin de protéger leur patrie. Par le silence, la foule soulignait symboliquement leur absence. À l'époque,

\footnotetext{
${ }^{24}$ GREGORY Adrian, The Silence of Memory: Armistice Day 1919-1946, op. cit., p. 226

${ }^{25} \mathrm{lbid}$.

${ }^{26}$ BRown Steven D., "Three minutes of silence : Social technologies of public commemoration », op. cit., p. 253.
} 
le patriotisme exacerbé par la guerre couplé au fait que rares étaient les Britanniques qui n'avaient pas perdu un proche fit en sorte que les Britanniques adhérèrent presque unanimement au projet des deux minutes de silence. Dès lors, c'est bien l'absence des commémorés qui a permis à chacun des individus composant la foule devant Whitehall de trouver une signification personnelle au silence.

À l'inverse, les formes récentes de commémoration par le silence ne reposent pas sur l'absence des commémorés ${ }^{27}$. Au gré des évolutions technologiques, il est devenu possible de filmer la foule lors des minutes de silence et même de projeter des images. Dès lors, progressivement, par le biais de photographies puis de montages vidéos, les commémorés sont réapparus lors des cérémonies. Ainsi, la recherche d'une signification personnelle de la part des participants a été remplacée par l'imposition d'une version uniformisée de l'événement commémoré. Si, pour le silence de l'Armistice, l'accent avait été mis sur le travail de compréhension et d'appropriation, on insiste désormais la plupart du temps sur l'aspect visuel, esthétique et émotionnel ${ }^{28}$.

\section{« Y être » versus « en être »}

Un autre élément permettant de différencier le silence qui s'inscrit dans les commémorations de l'Armistice au Royaume-Uni et la pause des trois minutes observée après les tsunamis en Asie est à rechercher dans la signification donnée au silence.

En effet, de nombreuses critiques ont été émises à l'encontre des trois minutes de silence en hommage aux victimes des raz-de-marée. Ces dernières ont fait naître l'idée que la pause observée à travers le monde n'était qu'un "cadeau " fait aux victimes des catastrophes en Asie. En utilisant la symbolique du "cadeau $»^{29}$, l'objectif était de dénoncer le fait qu'aucun discours ne venait justifier le pourquoi du silence observé.

À travers le monde, des individus se sont figés quelques instants de manière à former des monuments éphémères en hommages à des inconnus qui avaient vécu un désastre d'une ampleur difficilement inimaginable.

À l'inverse du silence de l'Armistice, les participants au silence des tsunamis n'étaient pas en mesure d'identifier une dimension personnelle à la souffrance commémorée.

${ }^{27}$ BRown Steven D., «Three minutes of silence : Social technologies of public commemoration ", op. cit., p. 253.

${ }^{28}$ Ibid., p. 252.

${ }^{29}$ Ibid., p. 259. 
Si on ajoute à cela l'absence de discours fédérateur, il est probable que les individus se soient interrogés durant trois minutes sur le sens à donner à leur participation, laissant l'objet réel de la commémoration non-abordé.

\section{Quand le silence devient l'unique étape du processus commémoratii}

Il y a une dernière explication qui permet de finir d'ancrer l'idée selon laquelle les manifestations récentes de commémorations par le silence sont une récupération de la forme de la pratique mais pas du fond.

Les commémorations de l'Armistice au Royaume-Uni, si elles s'articulent autour des deux minutes de silence, ne se résument pas à elles. En effet, il s'agit d'un assemblage complexe de discours et d'outils divers que sont le silence, le Cénotaphe de Whitehall ou encore le «Poppy Appeal "30. C'est cet « univers mémoriel » que le silence vient compléter qui permet à chaque Britannique, à la clôture des commémorations, de se sentir membre d'une communauté de destin.

À l'inverse, les usages récents du silence et, a fortiori, la pause des trois minutes en hommage aux victimes des tsunamis en Asie, ne sont pas renforcés par un tel système commémoratif. Pour la plupart, ils n'ont lieu qu'une seule fois et ne sont précédés ni suivis d'aucune forme de discours. Dès lors, il serait inutile de les doter d'une symbolique propre. C'est pour cette raison que les recours récents au silence empruntent le plus souvent à des répertoires déjà existants.

Les commémorations des attentats de Madrid en 2004 illustrent bien cet état de fait puisque ce sont les cloches des églises de la ville qui ont annoncé la fin du silence, donnant aux commémorations une dimension religieuse.

Ainsi, le silence de l'Armistice au Royaume-Uni s'inscrit dans un ensemble spécifique de techniques commémoratives. À chaque répétition, des discours affublent le silence d'une signification particulière pouvant évoluer selon les contextes politiques. Enfin, s'agissant d'un élément central du système commémoratif britannique, le silence continue à unir les Britanniques après que le bruit de la ville ait repris ses droits.

À l'inverse, les récentes commémorations par le silence obéissent davantage à une logique "on-off " ${ }^{31}$. Le silence devient l'unique étape du processus commémoratif. Les participants deviennent eux-mêmes

\footnotetext{
${ }^{30}$ BRown Steven D., «Three minutes of silence : Social technologies of public commemoration », op. cit., p. 248.
} 
les sujets de la commémoration. Trop occupés à rechercher le sens qu'ils donnent à ce qu'ils sont en train de faire, ils ne travaillent plus à remettre le passé en perspective. Après que le silence ait été rompu, ils n'y repenseront probablement plus jamais ${ }^{32}$.

Avant de conclure, un récapitulatif de la comparaison entre le silence de l'Armistice et celui observé en hommage aux victimes du tsunami peut s'avérer utile. Dans un souci de clarté, celui-ci est proposé sous la forme d'un tableau comparatif.

\begin{tabular}{|c|c|c|}
\hline & Silence Armistice & Silence Tsunami \\
\hline Durée & 2 minutes & 3 minutes \\
\hline Origine & Autorités & Autorités \\
\hline Répétition & Annuelle & Unique \\
\hline Commémorés & Absents & Présents \\
\hline Objectifs & Identification et partage & "Cadeau» \\
\hline Répertoires commémoratifs & Propre & Emprunté ou inexistant \\
\hline Passé & Remis en question & Non-abordé \\
\hline
\end{tabular}

Les développements qui précèdent semblent nous indiquer que l'outil commémoratif qu'est le silence peut, selon la façon dont il est mis en œuvre, renvoyer à des réalités très différentes. Pour cette raison, Steven Brown suggère que le silence devrait être remplacé par un autre outil lorsqu'il s'agit de commémorations de type "on-off " afin d'éviter toute confusion ${ }^{33}$. Parmi les pistes envisagées, il en est une qui commence à se répandre dans le milieu sportif : la commémoration par les applaudissements. Viendra peut-être un jour, plus ou moins proche, où afin de commémorer le passé, l'assistance, qui autrefois aurait gardé le silence pendant plusieurs minutes, lancera une salve d'applaudissements ${ }^{34}$.

\section{Conclusion}

L'objectif de cet article était de justifier les deux affirmations figurant dans son intitulé. D'une part, il s'agissait de démontrer que le silence peut être considéré comme un outil de construction mémorielle. D'autre part, il était question de faire état, par le biais d'un exer-

32 Brown Steven D., "Three minutes of silence : Social technologies of public commemoration ", op. cit., p. 260.

${ }_{33}$ Voy. Supra. 
cice de comparaison, des différences existant entre le silence de l'Armistice au Royaume-Uni et la pause de trois minutes observée en 2005 après les tsunamis en Asie. Enfin, ces développements devaient permettre de déterminer si le rôle du silence avait différé entre ces deux manifestations.

En ce qui concerne le silence comme outil de construction mémorielle, les développements qui précèdent semblent confirmer que le silence peut effectivement tenir ce rôle. Bien qu'à l'entame de ce travail, lorsque la notion de silence fut définie, le terme fut notamment décrit comme le fait de se taire, de ne pas parler, de ne pas tenir un discours, ce postulat est désormais à nuancer. Le silence, au même titre que le discours, peut être amené à intervenir dans un processus de construction mémorielle.

Si l'on s'intéresse maintenant à la distinction faite entre le silence de l'Armistice au Royaume-Uni et celui des tsunamis en Asie, le présent travail s'est efforcé de démontrer les nombreux aspects qui les différenciaient. Ainsi, tandis que le silence de l'Armistice se sert de l'absence des commémorés pour conduire les Britanniques à questionner le passé, les recours récents au silence semblent se limiter à la dimension esthétique de l'acte et vont jusqu'à brouiller la frontière entre acteurs et sujets des commémorations.

Le dernier objectif de ce travail était d'identifier si l' " outil silence ", malgré des contextes différents, avait conservé un rôle identique lors de ces deux manifestations. II apparaît que le silence, de la même façon que n'importe quel outil, ne peut entrer en action qu'à travers l'acte d'un individu, d'une communauté ou encore d'un gouvernement. Dès lors, cet acteur, quel qu'il soit, va déterminer le rôle du silence. Ainsi, les rôles du silence sont multiples et dépendent du cadre dans lequel ce dernier est mis en œuvre. Selon les cas, le silence permettra de susciter la réflexion à propos d'événements passés ou, au contraire, il ne sera qu'une marque d'empathie dénuée de toute remise en question.

Tous ces développements nous invitent à envisager l'inflation du silence avancée par Andy MCSMITH avec prudence car, si inflation du silence il y a, encore faut-il savoir de quel silence il s'agit. 